\title{
Accumulation of Toxic $\alpha$-Synuclein Oligomer within Endoplasmic Reticulum Occurs in $\alpha$-Synucleinopathy In Vivo
}

\author{
Emanuela Colla, ${ }^{1}$ Poul H. Jensen, ${ }^{2}$ Olga Pletnikova, ${ }^{1}$ Juan C. Troncoso, ${ }^{1}$ Charles Glabe, ${ }^{3}$ and Michael K. Lee ${ }^{1,4,5}$ \\ ${ }^{1}$ Department of Pathology, Johns Hopkins University School of Medicine, Baltimore, Maryland 21205, ${ }^{2}$ Department of Medical Biochemistry, Aarhus \\ University, Aarhus 8000, Denmark, ${ }^{3}$ Department of Molecular Biology and Biochemistry, University of California, Irvine, California 92697 , and \\ ${ }^{4}$ Department of Neuroscience and ${ }^{5}$ Institute for Translational Neuroscience, University of Minnesota, Minneapolis, Minnesota 55102
}

In Parkinson's disease (PD) and other $\alpha$-synucleinopathies, prefibrillar $\alpha$-synuclein $(\alpha S)$ oligomer is implicated in the pathogenesis. However, toxic $\alpha$ S oligomers observed using in vitro systems are not generally seen to be associated with $\alpha$-synucleinopathy in vivo. Thus, the pathologic significance of $\alpha \mathrm{S}$ oligomers to $\alpha \mathrm{S}$ neurotoxicity is unknown. Herein, we show that, $\alpha \mathrm{S}$ that accumulate within endoplasmic reticulum (ER)/microsome forms toxic oligomers in mouse and human brain with the $\alpha$-synucleinopathy. In the mouse model of $\alpha$-synucleinopathy, $\alpha \mathrm{S}$ oligomers initially form before the onset of disease and continue to accumulate with the disease progression. Significantly, treatment of $\alpha \mathrm{S}$ transgenic mice with Salubrinal, an anti-ER stress compound that delays the onset of disease, reduces ER accumulation of $\alpha \mathrm{S}$ oligomers. These results indicate that $\alpha \mathrm{S}$ oligomers with toxic conformation accumulate in ER, and $\alpha \mathrm{S}$ oligomerdependent ER stress is pathologically relevant for PD.

\section{Introduction}

Parkinson's disease (PD) is the second most common neurodegenerative disease after Alzheimer's disease (AD). While the etiology of PD is unknown in most cases, degenerating neuronal populations in PD exhibit $\alpha$-synuclein $(\alpha S)$ abnormalities and mutations in the $\alpha S$ gene cause familial PD, indicating that the $\alpha \mathrm{S}$ abnormalities are mechanistically linked to pathogenesis of PD and other $\alpha$-synucleinopathies (Vila and Przedborski, 2004; Obeso et al., 2010).

Neuropathological studies of human $\alpha$-synucleinopathy cases establish $\alpha$ S aggregates, appearing as Lewy bodies and neurites, as prominent pathological features. Further, transgenic (Tg) mouse models of $\alpha$-synucleinopathy with overt progressive neurodegeneration are associated with $\alpha \mathrm{S}$ aggregates. These results have supported view that the $\alpha$ S fibrils are of pathogenic importance in PD and other $\alpha$-synucleinopathies (Obeso et al., 2010). However, this view has been challenged due to inconsistent correlations between $\alpha \mathrm{S}$ fibrils and neurodegeneration in several model

\footnotetext{
Received Oct. 24, 2011; revised Dec. 9, 2011; accepted Dec. 20, 2011.

Author contributions: E.C. and M.K.L. designed research; E.C., O.P., and M.K.L. performed research; P.H.J., O.P., J.C.T., and C.G. contributed unpublished reagents/analytic tools; E.C. and M.K.L. analyzed data; E.C., P.H.J., and M.K.L. wrote the paper

This work was supported by NIH Grants NS038065, NS0380377, NS055776, and ES017384 (M.K.L.); and Lundbeck Foundation and European Community's Seventh Framework Programme (P.H.J.). We thank Dr. Virginia Lee for kindly providing the Syn 303 antibody.

The authors declare no conflict of interest.

This article is freely available online through the J Neurosci Open Choice option.

Correspondence should be addressed to Dr. Michael K. Lee, Department of Neuroscience, University of Minnesota, 2101 6th Street SE, Minneapolis, MN 55102.E-mail:mklee@umn.edu.

E. Colla's present address: Laboratory of Neurobiology, Scuola Normale Superiore, 56126 Pisa, Italy.

DOI:10.1523/JNEUROSCI.5368-11.2012

Copyright $\odot 2012$ the authors $\quad 0270-6474 / 12 / 323301-05 \$ 15.00 / 0$
}

systems (Volles and Lansbury, 2003). In addition, membranedisrupting and cytotoxic properties of various in vitro assembled $\alpha$ S oligomers and the emerging evidence for toxic $\mathrm{A} \beta$ oligomers in $\mathrm{AD}$ have fueled the view that prefibrillar soluble $\alpha \mathrm{S}$ oligomers may be pathogenic in PD (Volles and Lansbury, 2003; Lansbury and Lashuel, 2006). However, in vivo relevance of the soluble $\alpha \mathrm{S}$ oligomers in PD is uncertain due to the lack of convincing evidence for disease-linked accumulation of toxic soluble $\alpha \mathrm{S}$ oligomers. Thus, while multiple pathogenic mechanisms are proposed for PD based on the soluble oligomers (Obeso et al., 2010), it is unknown whether any of the proposed toxic mechanisms contribute to neurodegeneration associated with $\alpha$-synucleinopathies.

In this report, we show that $\alpha$-synucleinopathy in human and mouse is associated with preferential accumulation of toxic $\alpha \mathrm{S}$ oligomers within the endoplasmic reticulum/microsomes (ER/ $\mathrm{Ms}$ ). In the Tg mouse model of $\alpha$-synucleinopathy, accumulation of the toxic $\alpha S$ oligomers are temporally and spatially linked to the induction of chronic ER stress, neuropathology, and neurological abnormalities. Finally, Salubrinal, an anti-ER stress agent, attenuates disease manifestations and reduces accumulation of toxic $\alpha S$ oligomers in the $\alpha S$ Tg mouse model. We propose that toxic $\alpha \mathrm{S}$ oligomers accumulate in vivo and contribute to neurodegeneration by causing chronic ER stress response.

\section{Materials and Methods}

Transgenic mouse models. Transgenic mice expressing high levels of WT or mutant (A53T and $\mathrm{A} 30 \mathrm{P}) \alpha \mathrm{S}$ under the control of the mouse prion protein promoter have been described previously (Lee et al., 2002; Martin et al., 2006; Wang et al., 2008). Mice expressing A53T $\alpha$ S [line G2$3(A 53 T)$ ] develop fatal neurological disease at $\sim 12$ months of age. Presymptomatic mice were 10-14 months old and were free from any 

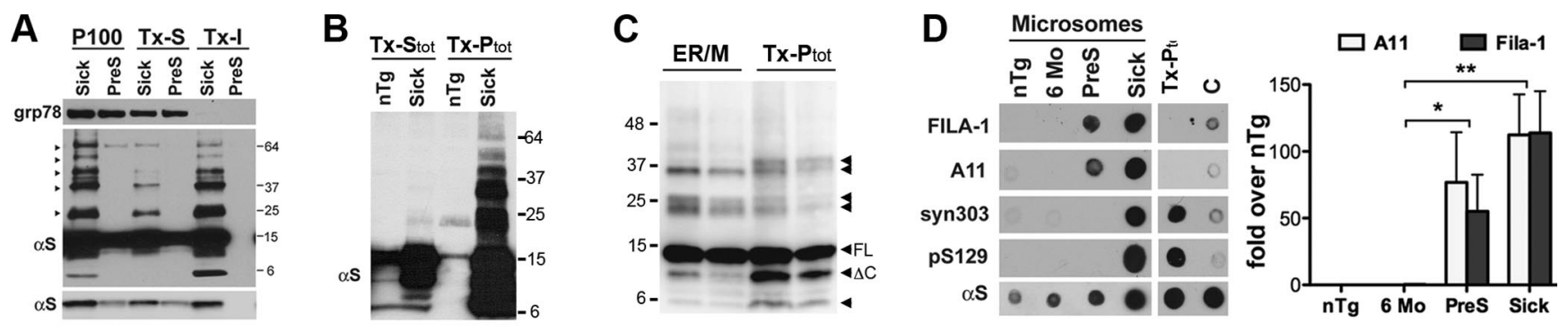
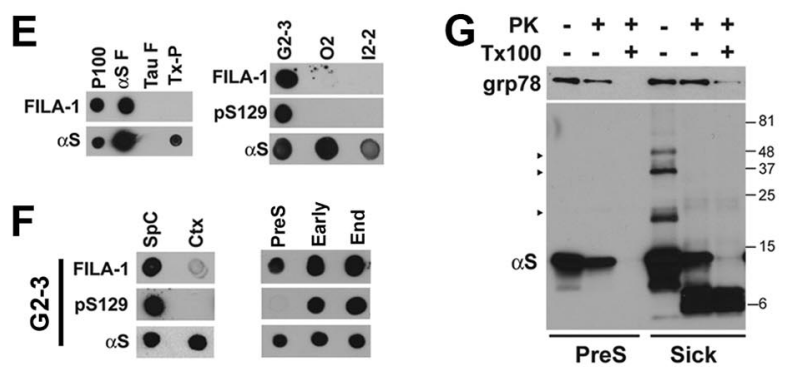
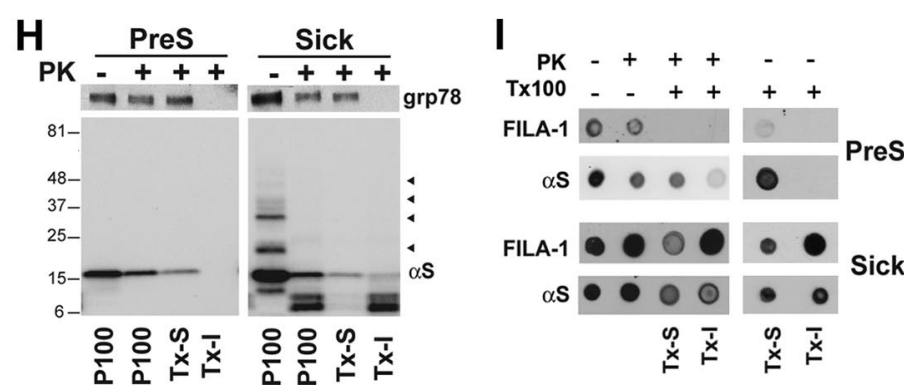

Figure 1. $\alpha S$ monomers and oligomers accumulate in the ER. A, ER/Ms (P100) obtained from PreS and end-stage (sick) A53T $\alpha S$ Tg mice were solubilized with $0.5 \%$ TX-100 and centrifuged to obtain the supernatant (TX-S) and the insoluble pellet (TX-I). Arrowheads, SDS-stable high-MW $\alpha$ S. Bottom, Shorter exposure of the $\alpha S$ blot. $B$, TX-S ${ }_{\text {tot }}$ and TX-P tot $_{\text {to }}$ fractions from the spinal cords of nTg and end-stage A53T $\alpha$ S Tg mice immunoblotted for $\alpha$ S. C, Detergent-insoluble fractions (TX-I from ER/M and TX-P tot) from two mice immunoblotted for $\alpha$ S. D, ER accumulation of toxic $\alpha S$ oligomers. Dot-blot analysis of SpC microsomes from nTg and A53T $\alpha$ S Tg mice (6 months, PreS, Sick). Also analyzed are total TX-P tot $_{\text {and }}$ C (detergent-free) from sick A53T $\alpha S$ Tg mice. Quantitative analysis of dot-blots showing values normalized for the total $\alpha$ S. Values are given as the mean $\pm \operatorname{SEM}(n=3-4) .{ }^{*} p<0.05,{ }^{* *} p<0.001$, one-way ANOVA. E, Left, Dot-blot analysis of microsomes from diseased $A 53 T$ mice (P100); in vitro assembled $\alpha \mathrm{S}(\alpha \mathrm{SF})$ and Tau (Tau-F) fibrils; or total insoluble fraction from Tg mice (TX-P tot $)$ shows FlLA-1 specificity for $\alpha S$ oligomers. Right, Dot-blots of various microsome fractions obtained from different $\alpha S$ Tg mouse lines [age-matched diseased $A 53 T \alpha S(G 2-3), A 30 P \alpha S\left(O_{2}\right)$, or WT $\left.\alpha S(I 2-2)\right]$. $F$, Dot-blots of ER/M from different areas $[\alpha-$ synucleinopathy-affected areas (SpC) or disease-free areas (Ctx). Right, Dot-blot analysis of ER/M from PreS or diseased (Early, End) mice for FILA-1 or $\alpha$ S. G, ER-associated $\alpha S$ monomer but not the high-MW $\alpha$ S is protected from proteolysis by PK. In the presence of $0.5 \%$ TX-100, both $\alpha$ S and the ER-resident grp78 are completely proteolyzed by PK. $\boldsymbol{H}$, ER/M fractions were first exposed to PK, followed by $0.5 \%$ TX-100 extraction, and were fractioned into TX-S and TX-I fractions. The $\alpha$ monomers remain soluble, but proteolyzed $\alpha$ fragments remain insoluble. $I$, Dot-blot analysis of ER/M fractions shown in $\boldsymbol{H}$ for FILA-1 or $\alpha$. In the PreS mice, the $\alpha$ S monomer and the oligomers are resistant to PK, indicating that they are in the ER lumen. Even in the absence of PK, TX-100 abolished both A11 and FILA-1 reactivity in PreS samples. I also shows dot-blot analysis of TX-100-soluble and -insoluble ER/M fractions without the prior PK treatment. Note that the FILA-1 signal is abolished with TX-100 in PreS mice but not in Sick mice.

motor dysfunction. All animal study methods were approved in full by the Institutional Animal Care and Use Committee of the Johns Hopkins University and were consistent with the requirements of the National Institutes of Health Office of Laboratory Animal Welfare Policy.

Subcellular fractionation. For subcellular fractionation of ER membrane-enriched microsomes, we used published methods (Cox and Emili, 2006) with modifications (Colla et al., 2012). To further enrich the ER content, the microsome preparation was purified using a discontinuous sucrose gradient (Croze and Morré, 1984). Pure mitochondria were obtained by discontinuous gradient fractionation of the crude mitochondria pellet (P10) (Vijayvergiya et al., 2005). Pure nuclei were isolated starting from the crude nuclei pellet (P1) (Cox and Emili, 2006) using a sucrose gradient (Colla et al., 2012).

Proteinase $K$ protection assay. The microsome fractions were treated with or without $50 \mu \mathrm{g} / \mathrm{ml}$ proteinase $\mathrm{K}$ (PK; Roche) and $1 \%$ Triton X-100 (TX-100) for $20 \mathrm{~min}$ on ice. The reaction was stopped by addiction of 2 $\mathrm{mm}$ (final concentration) phenylmethylsulfonyl fluoride.

Immunoblotting and dot-blot analysis. Immunoblot and dot-blot analysis of mice and human brain tissues were performed as previously described (Lee et al., 2002; Li et al., 2004, 2005; Martin et al., 2006; Wang et al., 2008). For dot-blot analysis, lysates were spotted directly on the nitrocellulose membrane and let it dry completely. Immunoreactivity was visualized using chemiluminescence detection (Pierce) after incubations with the appropriate horseradish peroxidase-conjugated secondary antibody, using a CCD-based Bio-Rad Molecular Imager ChemiDoc XRS+ System or X-ray films. The immunoreactive band intensities were determined by densitometry or the Quantity One software (Bio-Rad).

The following antibodies were used: grp78, grp94 (Stressgen); Syn-1, cytochrome C (BD Transduction Laboratories); A11 (Kayed et al., 2003); pser129- $\alpha$ S (Fujiwara et al., 2002); syn303 (Duda et al., 2000); and FILA-1 (Lindersson et al., 2004).

\section{Results}

Toxic $\alpha S$ oligomers in ER/Ms in A53T $\alpha S$ Tg mouse model of $\alpha$-synucleinopathy

Studies indicate that $\alpha \mathrm{S}$ can functionally impact multiple organelles (Gosavi et al., 2002; Smith et al., 2005; Cooper et al., 2006; Martinez-Vicente et al., 2008; Winslow et al., 2010). Similarly, our analysis of $\alpha \mathrm{S}$ species in various subcellular compartments (nuclear, mitochondrial, ER/M, and cytosol) reveals association of $\alpha \mathrm{S}$ monomers and high-molecular-weight (MW) $\alpha \mathrm{S}(\alpha \mathrm{S}$ aggregates), including serine-129 phosphorylated (pS129) $\alpha \mathrm{S}$ (Fujiwara et al., 2002), with ER/M (Colla et al., unpublished data). In the companion report (Colla et al., unpublished data), we also show that accumulation of $\alpha \mathrm{S}$ in the ER is linked to pathogenic chronic ER stress.

Because of the pathogenic relevance of ER associated $\alpha \mathrm{S}$ aggregates, the ER/M-associated $\alpha \mathrm{S}$ were examined in more detail. First, we examined whether the ER-associated $\alpha \mathrm{S}$ becomes detergent insoluble with disease, as we have shown for total $\alpha \mathrm{S}$ aggregates (Lee et al., 2002). The ER/M-associated $\alpha \mathrm{S}$ aggregates were fractionated into nonionic detergent $(0.5 \%$ TX-100)-soluble and detergent-insoluble fractions and subjected to the immunoblot analysis (Fig. 1A). The results show that the majority of SDSresistant high-MW $\alpha S$ variants in the ER/M from the sick A53T $\alpha S \mathrm{Tg}$ mice, as well as associated $\alpha \mathrm{S}$ monomers, pellet with detergent-insoluble (TX-I) fractions (Fig. 1A). Surprisingly, the detergent-soluble fractions (TX-S) of ER/M from the sick Tg mice contain significant amounts of SDS-resistant high-MW $\alpha \mathrm{S}$ variants (Fig. $1 \mathrm{~A}$ ). Similar analysis using total spinal cord lysates 
does not show soluble high-MW $\alpha \mathrm{S}$ variants (Fig. $1 B$ ). We also performed a side-by-side immunoblot comparison of the TX-I $\mathrm{ER} / \mathrm{M}$ and the total detergent-insoluble $\alpha \mathrm{S}$ (TX-P $\mathrm{P}_{\text {tot }}$ ) fractions from the end-stage $A 53 T \alpha S$ Tg mice (Fig. 1C). The results show that within each subcellular fraction the patterns of $\alpha \mathrm{S}$ variants are remarkably consistent between individuals. Further, despite some similarities, there were notable differences in the $\alpha \mathrm{S}$ variants between the fractions. In particular, compared with the TX- $\mathrm{P}_{\text {tot }}$, the ER/M TX-I fractions contain notably lower levels of truncated $\alpha \mathrm{S}$ variant (Fig. $1 C, \Delta \mathrm{C}$ ) and a less complex set of high-MW $\alpha$ S variants (Fig. $1 C$, arrowheads). While the $\alpha S$ variants in the ER/M represent a subset of the TX- $\mathrm{P}_{\text {tot }}$ variants, it is also clear that these two fractions contain biochemically distinct set of $\alpha \mathrm{S}$ variants. These results suggest that the ER/M-associated $\alpha S$ variants may be biochemically distinct from the total detergent-insoluble $\alpha \mathrm{S}$ variants (TX- $\left.\mathrm{P}_{\text {tot }}\right)$.

The soluble SDS-resistant $\alpha \mathrm{S}$ variants in ER/M could be the elusive "toxic-soluble oligomers." However, simple mobility shifts of $\alpha \mathrm{S}$ are not sufficient indicators of toxic conformation. Thus, the subcellular fractions were evaluated using antibodies to the toxic oligomers; A11, selective for all soluble toxic oligomers (Kayed et al., 2003), and FILA-1, selective for the toxic $\alpha \mathrm{S}$ oligomer/fibril (Lindersson et al., 2004; Paleologou et al., 2009). Both antibodies can also inhibit $\alpha \mathrm{S}$ oligomer-induced cytotoxicity in vitro (Kayed et al., 2003; Lindersson et al., 2004). As indicators of mature $\alpha \mathrm{S}$ aggregates, we also used antibodies that detect oxidized/aggregated $\alpha$ S (Syn303) (Duda et al., 2000) and pS129 $\alpha \mathrm{S}$ (Fujiwara et al., 2002).

Initial dot-blot analysis using FILA-1 shows that FILA-1 reacts with in vitro assembled $\alpha$ S fibrils (Fs) but does not bind to in vitro assembled tau Fs. Significantly, FILA-1 showed obvious reactivity to the ER/M fraction but not to the TX- $\mathrm{P}_{\text {tot }}$ from the A53T $\alpha S \mathrm{Tg}$ mice (Fig. $1 E$ ). To determine whether ER/M-associated toxic $\alpha S$ oligomers accumulate with disease in vivo, we performed dotblot analysis of ER/M, cytosolic (C), and total Triton-insoluble fractions (TX-P) from the spinal cords (SpC) of $A 53 T \alpha S$ Tg and nTg using the above antibodies (Fig. $1 D$ ). As expected, the cytosolic fraction exhibited robust signal for $\alpha \mathrm{S}$ but showed weak signals for all other antibodies. The TX- $\mathrm{P}_{\text {tot }}$ fractions reacted with antibodies characteristic for aggregated $\alpha \mathrm{S}$ (Syn303, pS129), confirming the presence of $\alpha \mathrm{S}$ aggregates, but did not react to antioligomer antibodies. In contrast, the ER/M from the aged presymptomatic (PreS) and diseased (sick) A53T $\alpha S$ Tg mice react with both A11 and FILA-1 (Fig. 1D). The A11 and FILA-1 reactions are not nonspecific cross-reactions to increased $\alpha \mathrm{S}$ levels since the TX-P and cytosolic fractions, despite the high levels of $\alpha \mathrm{S}$, do not react to either A11 or FILA-1. Similarly, the ER/M fraction from the mature (6-month-old) but pathology-free A53T $\alpha S$ Tg mice did not react to A11 or FILA-1. The FILA-1 reactivity to ER/M fraction is selective for the presence of $\alpha$-synucleinopathy as ER/Ms from aged $W T \alpha S(I 2-2)$ and $A 30 P \alpha S\left(\mathrm{O}_{2}\right)$ Tg mice are not FILA-1 reactive (Fig. $1 \mathrm{E}$ ). Similarly, ER/Ms from cortex of end-stage $A 53 T \alpha S$ Tg mice are not FILA-1 reactive (Fig. $1 F$ ). Collectively, these results show the disease specificity of toxic $\alpha \mathrm{S}$ oligomer accumulation.

The ER/M fractions from PreS were only reactive to antioligomer antibodies. However, the ER/M fractions from the sick A53T $\alpha$ S Tg mice also react to Syn303 and pS129 antibodies, indicating that these are mature aggregates (Fig. $1 D$ ). Similarly, analysis of PreS, early symptomatic, and end-stage mice show that the ER/M acquires $\mathrm{pS} 129 \alpha \mathrm{S}$ reactivity with the onset of motoric symptoms (Fig. $1 F$ ).

\section{Formation of toxic $\alpha S$ oligomers is initiated within the lumen of ER/M}

In our companion report, we showed that ER/M-associated $\alpha \mathrm{S}$ monomers are located within the lumen of ER/Ms. However, similar analysis of ER/Ms from symptomatic A53T $\alpha S$ Tg mice show that high-MW $\alpha \mathrm{S}$ variants are partially sensitive to PK, resulting in protected $\alpha \mathrm{S}$ monomer and proteolytic fragments of $\sim 6-8 \mathrm{kDa}$ (Fig. $1 G, H$ ). Significantly, when the PK-treated ER/M fractions were further separated into TX-S and TX-I fractions, most of the $\alpha \mathrm{S}$ monomer partitions with TX-S, while the $6 \mathrm{kDa} \mathrm{PK}$-derived $\alpha \mathrm{S}$ fragments remain insoluble with the TX-I (Fig. $1 \mathrm{H}$ ).

These results raise the possibility that while $\alpha \mathrm{S}$ monomers are located within the lumen, $\alpha \mathrm{S}$ oligomers could be forming on the cytosolic face of the ER/M. To study this possibility, we examined whether the ER/M-associated oligomers were sensitive to PK proteolysis. When the PK-treated ER/Ms were dot-blotted for FILA-1, the FILA-1-reactive oligomers were PK resistant in both presymptomatic and symptomatic Tg mice. Consistent with PK resistance of ER/M $\alpha \mathrm{S}$ (Fig. 1G,H), FILA-1 epitope in the presymptomatic mice is resistant to PK (Fig. $1 I$ ). Despite the partial PK proteolysis of high-MW $\alpha \mathrm{S}$ (Fig. 1G,H), FILA-1 epitopes survive both detergent and PK treatments (Fig. 1I). Based on the immunoblot analysis of the PK-digested fractions (Fig. $1 G, H$ ), we propose that the PK-resistant $\alpha \mathrm{S}$ fragment at $\sim 6 \mathrm{kDa}$ could be responsible for the oligomer conformation. This fragment is similar to the previously identified non-amyloid component regioncontaining peptide truncated at both the $\mathrm{N}$ and $\mathrm{C}$ termini ( $\mathrm{Li}$ et al., 2005). Collectively, the results suggest that $\alpha \mathrm{S}$ oligomers initially form within the ER/M lumen but become exposed to cytosol with the formation of detergent-stable $\alpha \mathrm{S}$ oligomers, a hypothesis consistent with the membrane destabilization properties of the toxic oligomers (Volles and Lansbury, 2003; Lansbury and Lashuel, 2006). Our data also indicate the presence of at least four distinct $\alpha \mathrm{S}$ pools in brain afflicted with $\alpha$-synucleinopathy: soluble monomers, A11/FILA-1-negative $\alpha$ S aggregates, soluble A11/ FILA-1 reactive (toxic) oligomers, and insoluble A11/FILA-1reactive (toxic) oligomers/aggregates.

Our studies also provide several additional insights. First, both A11 and FILA-1 reactivity is abolished if ER/Ms from PreS $\mathrm{Tg}$ mice are exposed to $0.5 \% \mathrm{TX}-100$, but when ER/Ms from symptomatic mice are used only A11 reactivity is abolished by TX-100. Thus, it is likely that the subset of $\alpha \mathrm{S}$ oligomers that reacts to both A11 and FILA-1 are labile to detergent exposure, such as in PreS Tg mice. However, because FILA-1 binds to both soluble oligomer and fibrils, FILA-1 epitope is associated with more mature $\alpha \mathrm{S}$ fibrils/oligomers in symptomatic mice. In conjunction with the temporal appearance of pS129 $\alpha$ S and syn303 reactivity, our results are consistent with the progressive conversion of the soluble $\alpha$ S oligomers to $\alpha$ S fibrils.

We also determined that $\alpha$ S oligomers accumulate in human $\alpha$-synucleinopathy. We examined human PD and control cases for the presence of toxic $\alpha \mathrm{S}$ oligomers (Fig. 2). While we used postmortem tissues, distribution of organelle markers upon fractionation show that our ER/M fractions are enriched for ER markers (calnexin), but are relatively free of mitochondrial and cytosolic markers (Colla et al., unpublished data). Dot-blot analysis ER/M fractions from human brain stem ( $\mathrm{BrSt})$, the area most affected by $\alpha$-synucleinopathy, show higher FILA- 1 and A11 reactivity in PD cases (Fig. $2 A$ ). Consistent with the advanced neuropathology in the human cases, the ER/M fractions from $\mathrm{PD}$ cases also show increased pS129 $\alpha \mathrm{S}$ reactivity (Fig. $2 A$ ) and highmolecular-mass $\alpha \mathrm{S}$ species (Fig. $2 A$ ). In contrast, total detergent- 
A

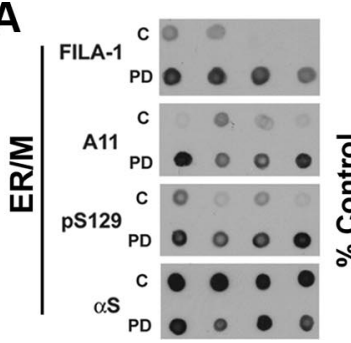

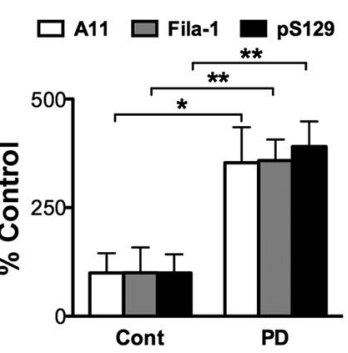

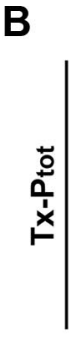

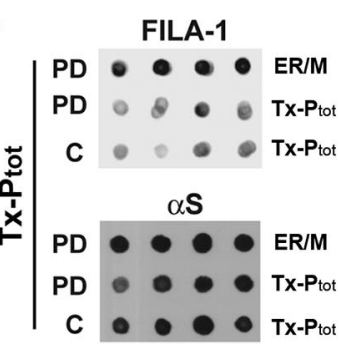

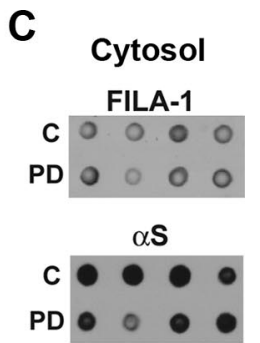

Figure 2. ER/M accumulation of $\alpha S$ oligomers in human PD cases. A, Dot-blot analysis of BrSt microsome fractions from human control and PD cases. Microsomes were probed with antibodies against $\alpha$ S oligomers and $\mathrm{pS} 129 \alpha$ S. Quantitative data values are normalized for total $\alpha$ S. Values are given as the mean \pm SEM $\left(n=8\right.$ for controls, $n=12$ for PD). ${ }^{*} p<0.05$, ${ }^{* *} p<0.01$, Student's $t$ test. $\boldsymbol{B}, \boldsymbol{C}$, Total $\alpha$ S aggregates (TX- $\mathrm{P}_{\text {tot }}$ ) and cytosolic fractions from PD brains do not show a significant accumulation of $\alpha S$ oligomers. TX-P $\mathrm{P}_{\text {tot }}(\boldsymbol{B})$ or cytosolic $(\boldsymbol{C})$ fractions from human PD cases were dot-blotted for FILA- 1 and $\alpha$ S. The ER/M fractions are also shown in $\boldsymbol{B}$.

soluble $\left(\right.$ TX-S tot ) and TX-P $\mathrm{P}_{\text {tot }}$ fractions from PD cases do not show increased levels of toxic oligomers (Fig. $2 B, C$ ). Thus, in both human and mouse cases of $\alpha$-synucleinopathy, there is selective accumulation of toxic $\alpha \mathrm{S}$ oligomers within the ER/M compartment. These results represent the first convincing demonstration of disease-associated in vivo accumulation of $\alpha \mathrm{S}$ oligomers with toxic conformations.

Salubrinal, an anti-ER stress compound, decreases the levels of ER/M-associated $\alpha S$ oligomers in the A53T $\alpha \mathrm{S} T g$ mouse model of $\alpha$-synucleinopathy

Consistent with the toxic nature of the ER-associated $\alpha \mathrm{S}$ oligomers, we were able to document ER dysfunction and activation of ER stress (ERS)/unfolded protein response with disease in the A53T $\alpha S \mathrm{Tg}$ mouse model (Colla et al., unpublished data). It is significant that the initial appearance of the toxic $\alpha S$ oligomers preceded the onset of ERS and motoric dysfunction in the mice. In addition, treatment of the $A 53 \mathrm{~T} \alpha \mathrm{STg}$ mouse model with Salubrinal, an anti-ERS compound (Boyce et al., 2005) that delays the onset of disease (Colla et al., unpublished data), leads to significantly reduced accumulation of toxic $\alpha \mathrm{S}$ oligomers (Fig. 3). This is a selective effect on the ER/M $\alpha \mathrm{S}$, as the total $\alpha S$ levels do not change with Salubrinal treatment (Colla et al., unpublished data). Overall, the results provide temporal, spatial, and quantitative associations between ER/M accumulation of $\alpha \mathrm{S}$ oligomer, ERS, and neurodegeneration.

\section{Discussion}

In this report, we show that a small subset of $\alpha \mathrm{S}$ localizes to the lumen of the ER/M (Colla et al. unpublished data) and accumulates as toxic $\alpha S$ oligomers. The initial appearance of the toxic $\alpha S$ oligomers precedes onset of disease and increases with the progression of $\alpha$-synucleinopathy. Moreover, treatment of mice with a known anti-ER stress agent, Salubrinal, attenuates disease manifestation and reduces levels of ER/M $\alpha \mathrm{S}$ oligomers. Overall, our results indicate the pathogenic significance of the ER/Massociated $\alpha \mathrm{S}$ oligomers and resulting ER stress in PD.

In $\mathrm{AD}$, in vivo accumulation of the toxic $\mathrm{A} \beta$ oligomers in disease conditions is well documented, and the pathologic significance of toxic $\mathrm{A} \beta$ oligomers in $\mathrm{AD}$ is generally accepted (Lansbury and Lashuel, 2006). However, for PD and other

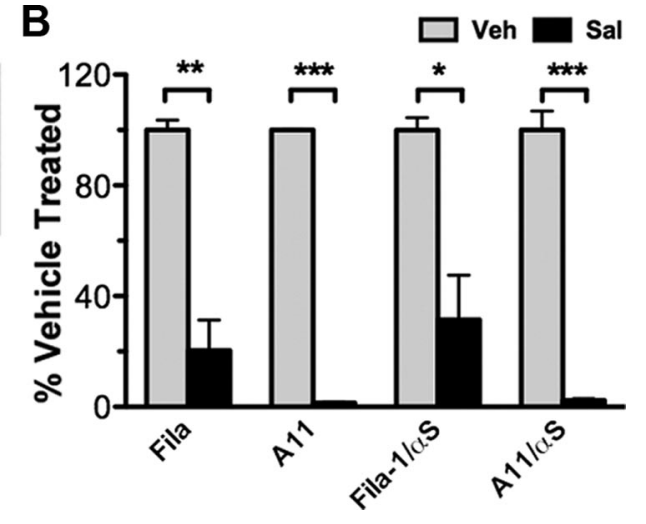

FILA-1

A11
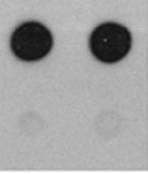

n of $\alpha$ S pathology. $\boldsymbol{A}$, ER/M fractions from Salubrinal (Sal)-treated A53T $\alpha$ S mice were dot-blotted for $\alpha$ S oligomers (A11, FILA-1). $\boldsymbol{B}$, The graphs showing the quantitative analysis of the dot-blots, which show significant decreases in $\alpha$ S oligomers with the Salubrinal treatment. The values were normalized either for total protein (FILA-1, A11) or for total $\alpha S$ (FILA-1/ $\alpha S, A 11 / \alpha S$ ) followed by the average of vehicle (Veh) group. Values are given as the mean \pm SEM $(n=3) .{ }^{* *} p<0.001,{ }^{* *} p<0.01,{ }^{*} p<0.05$, Student's $t$ test.

$\alpha$-synucleinopathies, that lack of evidence for in vivo accumulation of toxic $\alpha \mathrm{S}$ oligomers have limited the significance of toxic $\alpha \mathrm{S}$ oligomers in vivo. Thus, despite the in vitro studies showing cellular toxicity of $\alpha \mathrm{S}$ oligomers (Kayed et al., 2003; Lindersson et al., 2004; Tsika et al., 2010), the in vivo pathologic significance of $\alpha \mathrm{S}$ oligomers was unclear. In particular, while FILA-1- and A11reactive toxic $\alpha \mathrm{S}$ oligomers were seen using in vitro systems, evidence for in vivo accumulations of these types of $\alpha \mathrm{S}$ oligomers was limited (Paleologou et al., 2009). Thus, it is remarkable that we are able to show the accumulations of such oligomers in $\mathrm{Tg}$ mouse model and PD cases. The fact that the accumulation of ER/M-associated $\alpha \mathrm{S}$ oligomers was temporally and quantitatively linked to the onset of ER stress (Colla et al., 2012) and neurodegeneration supports the view that the ER/M $\alpha \mathrm{S}$ oligomers are indeed pathogenic. In this regard, the nonionic detergentsoluble $53 \AA \alpha$ S oligomers present in spinal cord of another A53T $\alpha S$ Tg mouse model (Tsika et al., 2010) may be related to the $\alpha S$ oligomer identified here.

Based on our results, we hypothesize a series of events leading to neurotoxicity. Normally, with aging and other stressed conditions, low levels of $\alpha \mathrm{S}$ in the ER forms immature soluble $\alpha \mathrm{S}$ oligomers that are A11/FILA-1 positive. These initial oligomers within the ER lumen may seed further assembly and maturation of $\alpha S$ oligomers, leading to accumulation of detergent-insoluble macro-aggregates/fibrils. Significantly, phosphorylation of Ser129 on $\alpha$ S seems to occur only with mature oligomers/aggregates, suggesting that this event is not required for the initiation of the toxic oligomer formation. Moreover, it is possible that the accu- 
mulation of $\alpha \mathrm{S}$ oligomers in the ER/M could be related to the secretion $\alpha \mathrm{S}$ and cell-to-cell transmission of $\alpha \mathrm{S}$ toxicity (Desplats et al., 2009). With the maturation of the $\alpha$ S oligomers from soluble to insoluble states, we hypothesize that the integrity of ER membranes is compromised and exposes portions of ER lumen to cytosolic compartment, leading to chronic ERS (shown in our companion report, Colla et al., 2012). While $\alpha$ S oligomers appear before ERS, it is also possible that onset of ERS promotes further accumulation and maturation of the toxic $\alpha$ S oligomer. While the mechanistic particulars behind our findings will require further studies, our results show that, in $\alpha$-synucleinopathy, toxic $\alpha$ S oligomer accumulation precedes disease onset and matures with disease progression. Thus, the results in this and our companion report support direct pathological links among $\alpha \mathrm{S}$ oligomers, ERS, and $\alpha$-synucleinopathy.

\section{References}

Boyce M, Bryant KF, Jousse C, Long K, Harding HP, Scheuner D, Kaufman RJ, Ma D, Coen DM, Ron D, Yuan J (2005) A selective inhibitor of eIF2alpha dephosphorylation protects cells from ER stress. Science 307:935-939.

Colla E, Coune P, Liu Y, Pletnikova O, Troncoso JC, Iwatsubo T, Schneider BL, Lee MK (2012) Endoplasmic reticulum stress is important for the manifestations of $\alpha$-synucleinopathy in vivo. J Neurosci 32:3306-3320.

Cooper AA, Gitler AD, Cashikar A, Haynes CM, Hill KJ, Bhullar B, Liu K, Xu K, Strathearn KE, Liu F, Cao S, Caldwell KA, Caldwell GA, Marsischky G, Kolodner RD, Labaer J, Rochet JC, Bonini NM, Lindquist S (2006) Alpha-synuclein blocks ER-Golgi traffic and Rab1 rescues neuron loss in Parkinson's models. Science 313:324-328.

Cox B, Emili A (2006) Tissue subcellular fractionation and protein extraction for use in mass-spectrometry-based proteomics. Nat Protoc $1: 1872-1878$

Croze EM, Morré DJ (1984) Isolation of plasma membrane, golgi apparatus, and endoplasmic reticulum fractions from single homogenates of mouse liver. J Cell Physiol 119:46-57.

Desplats P, Lee HJ, Bae EJ, Patrick C, Rockenstein E, Crews L, Spencer B, Masliah E, Lee SJ (2009) Inclusion formation and neuronal cell death through neuron-to-neuron transmission of alpha-synuclein. Proc Natl Acad Sci U S A 106:13010-13015.

Duda JE, Giasson BI, Chen Q, Gur TL, Hurtig HI, Stern MB, Gollomp SM, Ischiropoulos H, Lee VM, Trojanowski JQ (2000) Widespread nitration of pathological inclusions in neurodegenerative synucleinopathies. Am J Pathol 157:1439-1445.

Fujiwara H, Hasegawa M, Dohmae N, Kawashima A, Masliah E, Goldberg MS, Shen J, Takio K, Iwatsubo T (2002) alpha-Synuclein is phosphorylated in synucleinopathy lesions. Nat Cell Biol 4:160-164.

Gosavi N, Lee HJ, Lee JS, Patel S, Lee SJ (2002) Golgi fragmentation occurs in the cells with prefibrillar alpha-synuclein aggregates and precedes the formation of fibrillar inclusion. J Biol Chem 277:48984-48992.

Kayed R, Head E, Thompson JL, McIntire TM, Milton SC, Cotman CW, Glabe CG (2003) Common structure of soluble amyloid oligomers implies common mechanism of pathogenesis. Science 300:486-489.

Lansbury PT, Lashuel HA (2006) A century-old debate on protein aggregation and neurodegeneration enters the clinic. Nature 443:774-779.
Lee MK, Stirling W, Xu Y, Xu X, Qui D, Mandir AS, Dawson TM, Copeland NG, Jenkins NA, Price DL (2002) Human alpha-synuclein-harboring familial Parkinson's disease-linked Ala-53 $\rightarrow$ Thr mutation causes neurodegenerative disease with alpha-synuclein aggregation in transgenic mice. Proc Natl Acad Sci U S A 99:8968-8973.

Li W, Lesuisse C, Xu Y, Troncoso JC, Price DL, Lee MK (2004) Stabilization of $\alpha$-synuclein protein with aging and familial Parkinson's disease-linked A53T mutation. J Neurosci 24:7400-7409.

Li W, West N, Colla E, Pletnikova O, Troncoso JC, Marsh L, Dawson TM, Jäkälä P, Hartmann T, Price DL, Lee MK (2005) Aggregation promoting C-terminal truncation of alpha-synuclein is a normal cellular process and is enhanced by the familial Parkinson's disease-linked mutations. Proc Natl Acad Sci U S A 102:2162-2167.

Lindersson E, Beedholm R, Højrup P, Moos T, Gai W, Hendil KB, Jensen PH (2004) Proteasomal inhibition by alpha-synuclein filaments and oligomers. J Biol Chem 279:12924-12934.

Martin LJ, Pan Y, Price AC, Sterling W, Copeland NG, Jenkins NA, Price DL, Lee MK (2006) Parkinson's disease $\alpha$-synuclein transgenic mice develop neuronal mitochondrial degeneration and cell death. J Neurosci 26:41-50.

Martinez-Vicente M, Talloczy Z, Kaushik S, Massey AC, Mazzulli J, Mosharov EV, Hodara R, Fredenburg R, Wu DC, Follenzi A, Dauer W, Przedborski S, Ischiropoulos H, Lansbury PT, Sulzer D, Cuervo AM (2008) Dopamine-modified alpha-synuclein blocks chaperone-mediated autophagy. J Clin Invest 118:777-788.

Obeso JA, Rodriguez-Oroz MC, Goetz CG, Marin C, Kordower JH, Rodriguez M, Hirsch EC, Farrer M, Schapira AH, Halliday G (2010) Missing pieces in the Parkinson's disease puzzle. Nat Med 16:653-661.

Paleologou KE, Kragh CL, Mann DM, Salem SA, Al-Shami R, Allsop D, Hassan AH, Jensen PH, El-Agnaf OM (2009) Detection of elevated levels of soluble alpha-synuclein oligomers in postmortem brain extracts from patients with dementia with Lewy bodies. Brain 132:1093-1101.

Smith WW, Jiang H, Pei Z, Tanaka Y, Morita H, Sawa A, Dawson VL, Dawson TM, Ross CA (2005) Endoplasmic reticulum stress and mitochondrial cell death pathways mediate A53T mutant alpha-synuclein-induced toxicity. Hum Mol Genet 14:3801-3811.

Tsika E, Moysidou M, Guo J, Cushman M, Gannon P, Sandaltzopoulos R, Giasson BI, Krainc D, Ischiropoulos H, Mazzulli JR (2010) Distinct region-specific $\alpha$-synuclein oligomers in A53T transgenic mice: implications for neurodegeneration. J Neurosci 30:3409-3418.

Vijayvergiya C, Beal MF, Buck J, Manfredi G (2005) Mutant superoxide dismutase 1 forms aggregates in the brain mitochondrial matrix of amyotrophic lateral sclerosis mice. J Neurosci 25:2463-2470.

Vila M, Przedborski S (2004) Genetic clues to the pathogenesis of Parkinson's disease. Nat Med [10 Suppl]:S58-S62.

Volles MJ, Lansbury PT Jr (2003) Zeroing in on the pathogenic form of alpha-synuclein and its mechanism of neurotoxicity in Parkinson's disease. Biochemistry 42:7871-7878.

Wang J, Martin E, Gonzales V, Borchelt DR, Lee MK (2008) Differential regulation of small heat shock proteins in transgenic mouse models of neurodegenerative diseases. Neurobiol Aging 29:586-597.

Winslow AR, Chen CW, Corrochano S, Acevedo-Arozena A, Gordon DE, Peden AA, Lichtenberg M, Menzies FM, Ravikumar B, Imarisio S, Brown S, O'Kane CJ, Rubinsztein DC (2010) alpha-Synuclein impairs macroautophagy: implications for Parkinson's disease. J Cell Biol 190:10231037. 